\title{
Capacity Analysis of the VBLAST MIMO System in Fading Condition with M-QAM Modulation
}

\author{
Samarendra Nath Sur \\ Sikkim Manipal Institute of Technology \\ Majitar, Rangpo, East Sikkim \\ Sikkim-737132, India
}

\author{
Rabindranath Bera \\ Sikkim Manipal Institute of Technology \\ Majitar, Rangpo, East Sikkim \\ Sikkim-737132, India
}

\begin{abstract}
Multiple input multiple output (MIMO) system using multiple transmit and receive antennas are widely recognized as the vital breakthrough that will allow future wireless systems to achieve higher data rates and also to increase diversity to combat channel fading. Therefore, a MIMO system can provide capacity gain and diversity gain. In this paper, we investigate the channel capacity issue of the Vertical-Bell Laboratories Layered Space-Time (VBLAST) multiple-input multiple-output (MIMO) system with minimum min square error (MMSE) receiver and zero-forcing (ZF) receivers in fading channel condition. In this paper, the difference in capacity of MIMO system with the MMSE receiver and $\mathrm{ZF}$ receiver is validated by comparing the mathematical relation and the simulated results.
\end{abstract}

\section{Keywords}

MIMO, MMSE, ZF, Capacity, Rician factor.

\section{INTRODUCTION}

Multiple-input-multiple-output (MIMO) technologies are extensively explored and accepted as one of the leading technology to support requirements of the user in future generation. From the perspective of the broad band communication scenario, the wireless communication technology should support a robust, reliable and very high speed communication link. Through the extensive research it has been highly recognized that MIMO have the potential to increase the (Shannon) capacity of the modern wireless systems [1] [2] [3]. Many schemes have been proposed to exploit the high spectral efficiency of MIMO channels, among which V-BLAST (as shown in figure 1) [4] [5] is relatively simple to implement and can provide high spectral efficiency. It has been shown in [1], [6] that when the receiver has access to the instantaneous channel gains, called the channel state information (CSI) [7], the capacity of a Rayleigh distributed at fading channel will increase almost linearly with the minimum of the number of transmit and receive antennas in comparison to that of a single input single out put (SISO) system. Lots of researches are going on the capacity analysis of multiple antenna systems in the Rayleigh distributed fading channel. Rayleigh models are realistic for environments with a large number of scatterers. However, in this paper we have analyzed the capacity of the channel in Rician fading environment, since both additive white gaussian noise (AWGN) and Rayleigh fading channels may be considered to be the limiting cases of the Rician channel, but Rician fading is also known to be a better model for wireless environments with a strong direct Line-Of-Sight (LOS) path [8] relative to the multipath components [9] [10]. The sum of a constant amplitude direct signal and a Rayleigh distributed scattered signal results in a signal with a Rician envelope distribution. The pdf of the Rician random variable $\mathrm{b}$ is given by

$$
p(b)= \begin{cases}2 b(1+k) e^{-k-(1+k) b^{2}} I_{0}(2 b \sqrt{k(k+1)}) & b \geq 0 \\ 0 & b<0\end{cases}
$$

where $\mathrm{K}$ is the Rician factor, denoting the power ratio of the direct and the scattered signal components.

For $\mathrm{K}=0$, there is no direct signal component and the Rician pdf becomes a Rayleigh pdf. On the other hand, large values of $\mathrm{K}$ indicate a slightly faded channel. For K approaching infinity, there is no fading at all resulting in an AWGN channel. And also in this paper, we present an in-depth capacity analysis [11] of the performance of the zero forcing (ZF) and minimum mean squared error (MMSE) equalizers applied to the Rician channel [12]. The linear ZF and MMSE equalizers [13] are classic functional blocks and are ubiquitous in digital communications [14]. They are also the building blocks of more advanced communication schemes such as the decision feedback equalizer (DFE).

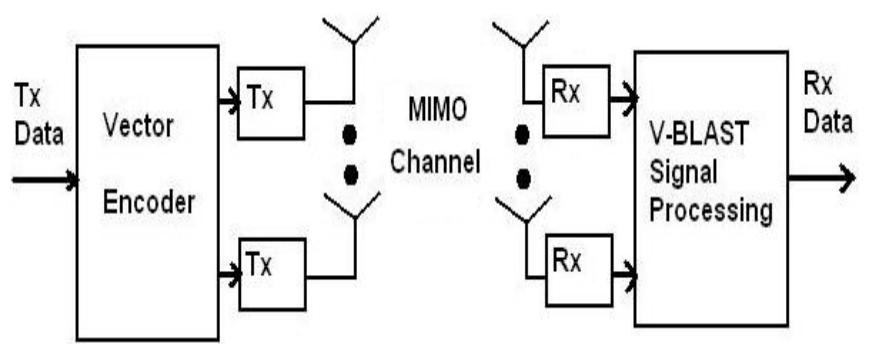

Figure 1. VBLAST System diagram

\section{MATHEMATICAL MODEL}

In this section, we analyze the capacity of MIMO channel in Rician fading environment. Let $\mathrm{M}$ and $\mathrm{N}$ be the number of transmitter and receiver antennas. Define the signal transmitted via the $M^{\text {th }}$ transmit antenna as $s_{M}(t)$ and $s(t)=\left[s_{1}(t) s_{2}(t) \ldots s_{M}\right.$ $(t)] T$. Then the received signal vector $\mathbf{r}$ can be written as

$\mathbf{r}=\mathbf{H s}+\mathbf{n}$

Where $\mathrm{H}$ is the channel matrix with dimension $\mathrm{MxN}, \mathrm{n}$ is the noise vector of dimension $\mathrm{Nx} 1$ and $\mathrm{r}$ is of $\mathrm{Nx} 1$. Now in presence of a Rician channel, which consist of a LOS component and NLOS component, the channel matrix is modeled as the weighted 
sum of Los component and the NLOS component. Therefore the channel matrix can be expressed as

$$
\mathrm{H}=\sqrt{\frac{\mathrm{k}}{\mathrm{k}+1}} \mathrm{H} \text { LOS }+\sqrt{\frac{1}{\mathrm{k}+1}} \mathrm{H} \text { NLOS }
$$

Where $\mathrm{k}$ denotes the Rician factor, which is the power ratio of a LOS component to NLOS components and $\mathrm{H}_{\mathrm{LOS}}$ is the channel matrix for the LOS propagation with no scattering and $\mathrm{H}_{\mathrm{NLOS}}$ is the channel matrix for the case with scattering only.

In matrix form, the equation 1 can be written as

$$
\left(\begin{array}{l}
\mathrm{r}_{1} \\
\mathrm{r}_{2} \\
\cdot \\
\cdot \\
\mathrm{r}_{\mathrm{N}}
\end{array}\right)=\left[\begin{array}{llll}
\mathrm{h}_{11} & \ldots \ldots \ldots . . . & . \mathrm{h}_{1, \mathrm{M}} \\
\cdot & & & \\
\cdot & & & \\
\mathrm{h}_{\mathrm{N}} & , 1 \ldots \ldots . & \mathrm{h}_{\mathrm{N}} & , \mathrm{M}
\end{array}\right]\left[\begin{array}{l}
\mathrm{s}_{1} \\
\mathrm{~s}_{2} \\
\cdot \\
\cdot \\
\mathrm{s}_{\mathrm{M}}
\end{array}\right]+\left[\begin{array}{l}
\mathrm{n}_{1} \\
\mathrm{n}_{2} \\
\cdot \\
\cdot \\
\mathrm{n}_{\mathrm{N}}
\end{array}\right]
$$

Now, the capacity of a MIMO system is given by the equation as follows

$$
c=\log 2 \operatorname{det}\left(I_{N}+\frac{1}{\sigma_{n}^{2}}\left(H \phi H^{H}\right)\right)
$$

Where $\mathrm{H}^{\mathrm{H}}$ is the Hermitian transpose of the matrix $\mathrm{H}, \phi$ is the covariance matrix of the transmitted signal $s(t)$ and $\sigma^{2} n$ variance of the noise vector. The covariance matrix $\phi$ can be defined as

$\varphi=\frac{\mathrm{p}}{\mathrm{n}} \mathrm{I}_{\mathrm{N}}$

Where $\mathrm{P}$ is the total transmitted power.

Now, the capacity of a MIMO channel can written as

$\mathrm{c}=\log _{2} \operatorname{det}\left(\mathrm{I}_{\mathrm{N}}+\mathrm{SNR} \cdot\left(\mathrm{H} \cdot \mathrm{H}^{\mathrm{H}}\right)\right)$

Where SNR is the average signal to noise ratio at each receiver antenna.

\section{CAPACITY analysis of linear detector system}

The capacity of a sub-channel in a MIMO system with linear detector (LD) can be written as

$$
\mathrm{c}_{\mathrm{LD}}=\log _{2}\left(\mathrm{I}_{\mathrm{N}}+\mathrm{SINR}\right)
$$

For ZF

If $\mathrm{F}_{\mathrm{ZF}}$ represent the transformation function corresponding to the ZF diversity combiner, then the capacity associated with the channel can be written as

$$
\mathrm{c}_{\mathrm{ZF}}=\log _{2}\left(1+\frac{\mathrm{SNR}}{\mathrm{F}_{\mathrm{ZF}}}\right)
$$

Now for $\mathrm{ZF}$ receiver $\mathrm{F}_{\mathrm{ZF}}=\left(\mathrm{H}^{\mathrm{H}} . \mathrm{H}\right)^{-1}$

$$
\mathrm{C}_{\mathrm{ZF}}=\log _{2}\left(1+\frac{\mathrm{SNR}}{\left(\mathrm{H}^{\mathrm{H}} \cdot \mathrm{H}\right)^{-1}}\right)
$$

Similarly for MMSE receiver

$$
\mathrm{F}_{\mathrm{M}}=\left(\mathrm{I}_{\mathrm{N}}(\mathrm{SNR})^{-1}+\mathrm{H}^{\mathrm{H}} \cdot \mathrm{H}\right)^{-1} \cdot \mathrm{H}^{\mathrm{H}}
$$

and after the application of $\mathrm{F}_{\mathrm{M}}$ on the received data, the SINR can be obtained as per the equation given below-

$$
\begin{aligned}
& \text { SINR }=\frac{1}{\left(I_{N}+(S N R) \cdot H^{H} \cdot H\right)^{-1}}-1 \\
& \Rightarrow \text { SINR }+1=\frac{1}{\left(I_{N}+(S N R) \cdot H^{H} \cdot H\right)^{-1}}
\end{aligned}
$$

Therefore

$$
\mathrm{C}_{\text {MMSE }}=\log _{2}\left(\frac{1}{\left(\mathrm{I}_{\mathrm{N}}+(\mathrm{SNR}) \cdot \mathrm{H}^{\mathrm{H}} \cdot \mathrm{H}\right)^{-1}}\right)-
$$

Now, MMSE based detector achieves higher SNR in comparison to that of a ZF based detector since minimize the mean square error (MSE) is equivalent to maximizing SINR. Thus the capacity with MMSE based detector is higher than that with ZF based detector.

Then $\mathrm{C}_{\text {MMSE}}-\mathrm{C}_{\mathrm{ZF}}$

$$
\begin{aligned}
& =\log _{2}\left(\frac{1}{\left(\mathrm{I}_{\mathrm{N}}+(\mathrm{SNR}) \cdot \mathrm{H}^{\mathrm{H}} \cdot \mathrm{H}\right)^{-1}}\right)-\log _{2}\left(1+\frac{\mathrm{SNR}}{\left(\mathrm{H}^{\mathrm{H}} \cdot \mathrm{H}\right)^{-1}}\right) \\
& =\log _{2}\left(\frac{1}{\left(\mathrm{I}_{\mathrm{N}}+(\mathrm{SNR}) \cdot \mathrm{H}^{\mathrm{H}} \cdot \mathrm{H}\right)^{-1} *\left(1+\frac{\mathrm{SNR}}{\left(\mathrm{H}^{\mathrm{H}} \cdot \mathrm{H}\right)^{-1}}\right)}\right)-(12)
\end{aligned}
$$

For large SNR, $\left(\mathrm{I}_{\mathrm{N}}+(\mathrm{SNR}) \cdot \mathrm{H}^{\mathrm{H}} \cdot \mathrm{H}\right)^{-1}$ can be expanded as follows

$$
\begin{aligned}
& =\left(\mathrm{SNR} \cdot \mathrm{H}^{\mathrm{H}} \cdot \mathrm{H}\right)^{-1}\left[\begin{array}{c}
1-\mathrm{I}_{\mathrm{N}}\left(\mathrm{SNR} \cdot \mathrm{H}^{\mathrm{H}} \cdot \mathrm{H}\right)^{-1}+ \\
\left\{\mathrm{I}_{\mathrm{N}}\left(\mathrm{SNR} \cdot \mathrm{H}^{\mathrm{H}} \cdot \mathrm{H}\right)^{-1}\right\}^{2}-\ldots .
\end{array}\right] \\
& =\left(\mathrm{SNR} \cdot \mathrm{H}^{\mathrm{H}} \mathrm{H}\right)^{-1}-\left[\mathrm{I}_{\mathrm{N}}\left(\mathrm{SNR} \cdot \mathrm{H}^{\mathrm{H}} \cdot \mathrm{H}\right)^{-2}\right]+\mathrm{O}(\mathrm{SNR})^{-3}
\end{aligned}
$$

Thus for large SNR level the equation 12 can be written as

$$
\begin{gathered}
=\log _{2}\left(\frac{1}{\left[\left(\mathrm{SNRH}^{\mathrm{H}} \cdot \mathrm{H}\right)^{-1}-\left\{\mathrm{I}_{\mathrm{N}}\left(\mathrm{SNRH} \mathrm{H}^{\mathrm{H}} \cdot \mathrm{H}\right)^{-2}\right\}+\mathrm{O}(\mathrm{SNR})^{-3}\right] \cdot\left(1+\frac{\mathrm{SNR}}{\left(\mathrm{H}^{\mathrm{H}} \cdot \mathrm{H}\right)^{-1}}\right)}\right) \\
=\log _{2}(\mathrm{SNR})-\log _{2}\left\{1-(\mathrm{SNR})^{-1} \mathrm{I}_{\left.\mathrm{N} \cdot\left(\mathrm{H}^{\mathrm{H}} \cdot \mathrm{H}\right)^{-1}+\mathrm{O}(\mathrm{SNR})^{-2}\right\}}^{-\log _{2}\left[\mathrm{SNR}+\left(\mathrm{H}^{\mathrm{H}} \cdot \mathrm{H}\right)^{-1}\right]}\right.
\end{gathered}
$$




\section{SIMULATION RESULTS}

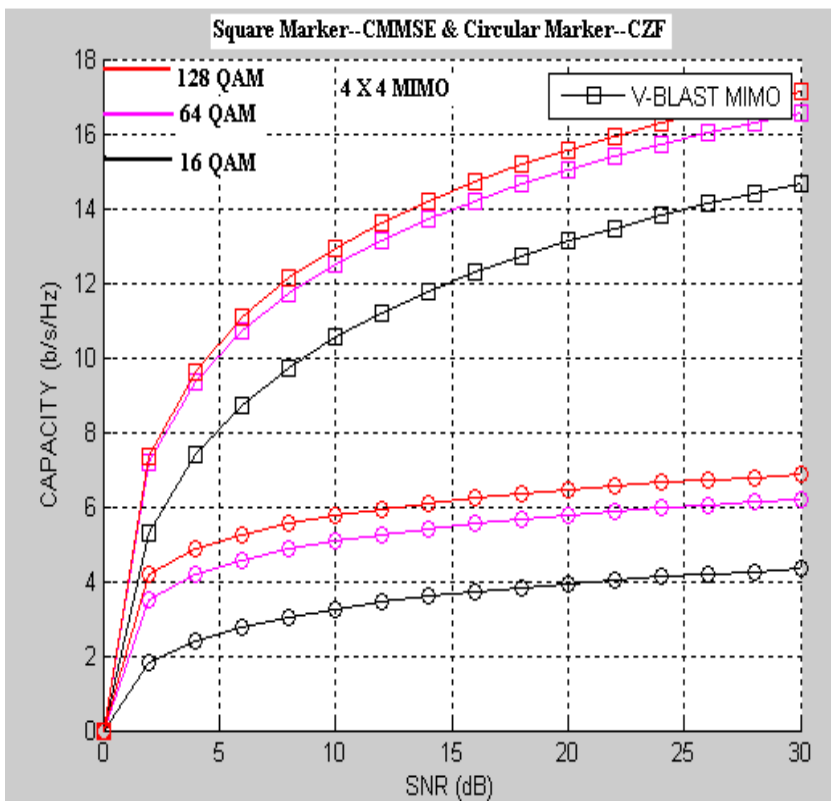

Figure 2: Capacity Vs SNR curves with MMSE and ZF receiver for different M-array QAM

Figure 2 shows the Capacity Vs SNR curves with different Marray QAM in Rician fading condition (with Rician factor, $\mathrm{k}=1$ ) for a $4 \mathrm{x} 4 \mathrm{MIMO}$ system. As in figure with the increase in the order of modulation i.e. 16QAM $\rightarrow 64$ QAM $\rightarrow$ 128QAM, the channel capacity increase consequently. Also as in figure, it is clear that with the increase in the SNR level the difference in channel capacity, corresponding to the MMSE receiver and the ZF receiver increases considerably i.e. $\mathrm{C}_{\mathrm{MMSE}}>\mathrm{C}_{\mathrm{ZF}}$ at the high SNR level.

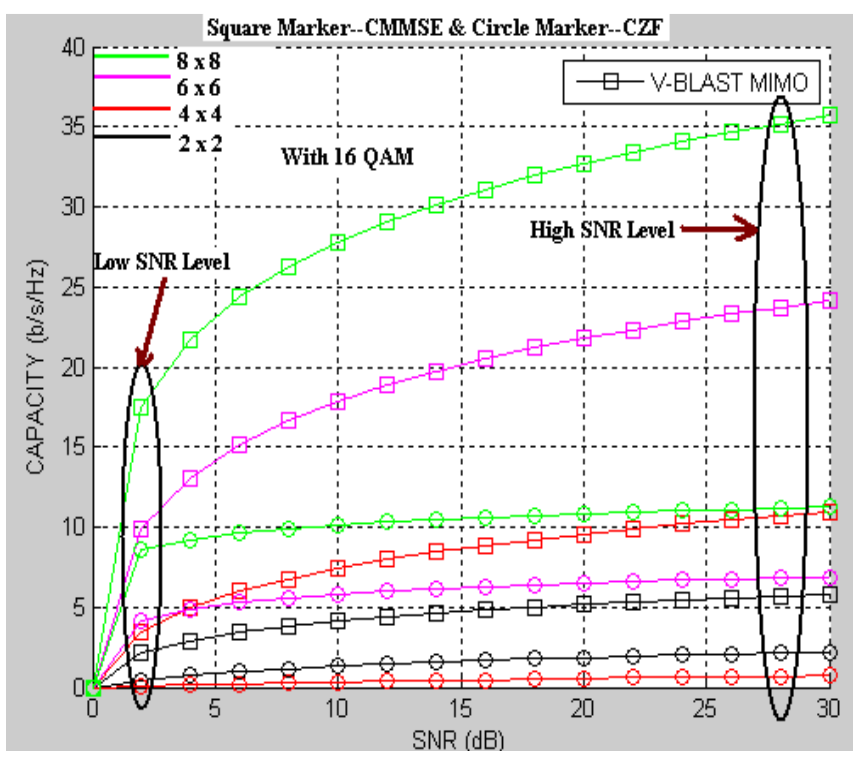

Figure 3: Capacity Vs SNR curves (with MMSE and ZF receiver) for different configuration of transmitter and receiver antenna in MIMO system.
Relative Comparision In Channel Capacity

a Series1 ..... Difference in capacity (CMMSE - CZF) at Low SNR Level of $2 \mathbf{d B}$ a Series2 -----Difference in capacity (CMMSE - CZF) at High SNR Level of $\mathbf{2 8} \mathbf{d B}$

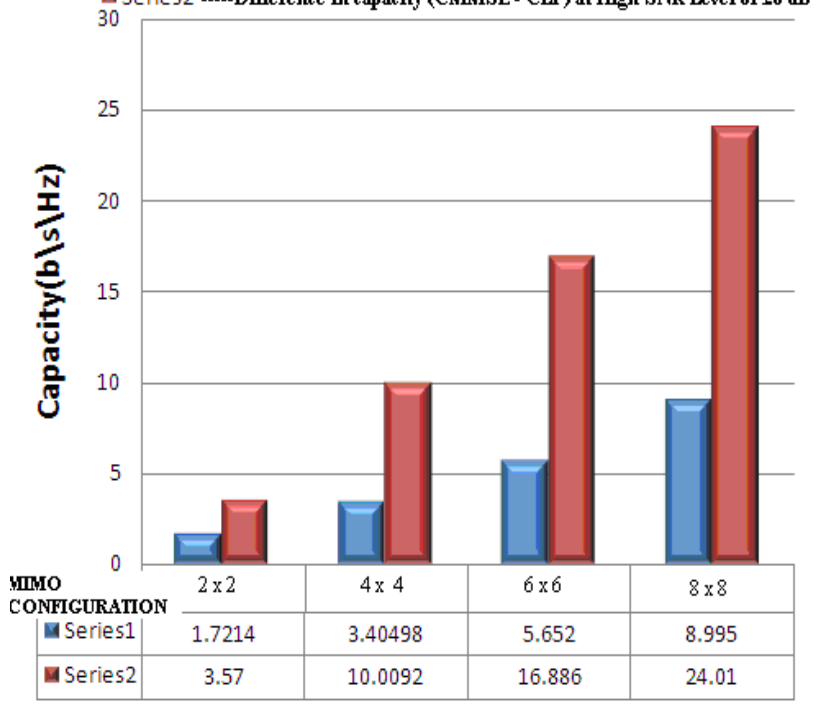

Figure 4: Difference in Capacity $\left(C_{M M S E}-C_{Z F}\right)$ for different configuration of MIMO system for two SNR level.

Figure 3 represent a comparative study on the channel capacity for different configuration of MIMO system. From figure it is clear that with the increase in number of transmitter and receiver antennas the channel capacity increases accordingly. Figure 4 shows the relative compression of the channel capacity with MMSE and ZF detector at the receiver side for two different SNR level (Low SNR level of $2 \mathrm{~dB}$ and high SNR level of $28 \mathrm{~dB}$ ). In figure 4 blue and red bars represent the channel capacity difference with MMSE and ZF receiver at the low and high SNR level respectively. As in figure at the high SNR level condition the capacity difference with MMSE and ZF receiver is large in comparison to that at low SNR level i.e. at high SNR level condition $\mathrm{C}_{\mathrm{MMSE}}$ is much greater than $\mathrm{C}_{\mathrm{ZF}}$.

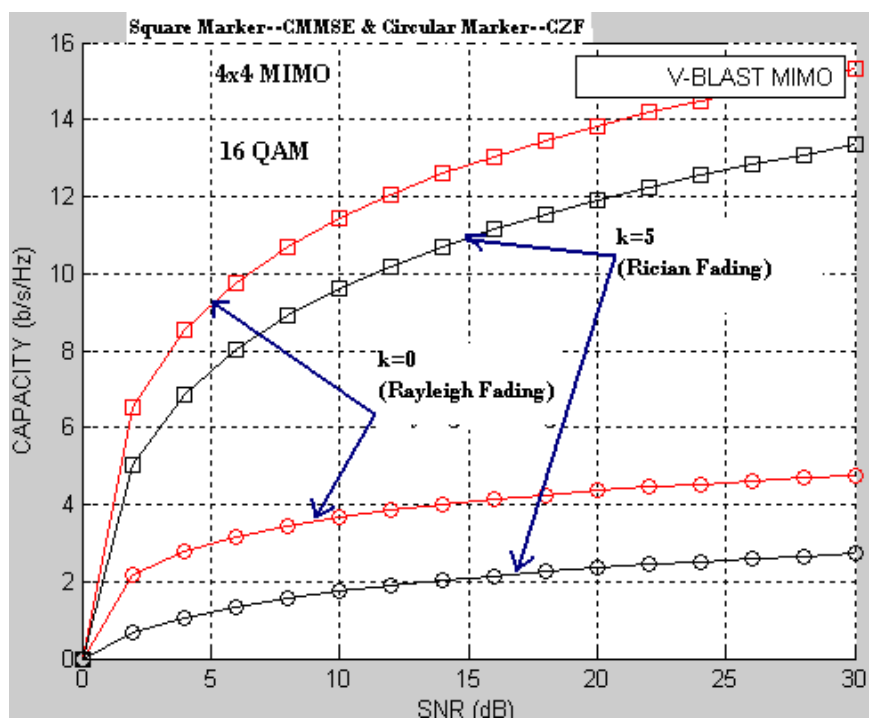

Figure 5.The channel capacity as a function of SNR for $4 \times 4$ MIMO system (with MMSE and ZF receiver) with Rayleigh and Rician channels. 
Figure 5 represent the comparative study regarding the variation of channel capacity with the variation in Rician $\mathrm{k}$ factor. For all values of SNR, as the value of $\mathrm{K}$ increases, the channel capacity decreases. This is because the increase in $\mathrm{K}$ factor emphasizes the deterministic part of the channel or in other word for the fixed antenna separation; the other source of channel correlation is from the Rician factor. Therefore as the deterministic channel is of rank 1 and so the capacity decreases. Thus, the capacity with $K=5 \mathrm{~dB}$ shown in the figure is lower than that with $\mathrm{K}=0 \mathrm{~dB}$.

\section{CONCLUSION}

In this paper we have investigated the channel capacity of VBLAST MIMO system in fading channel condition with two different suboptimal linear receiver systems: MMSE and ZF algorithm based receiver. From our analysis we have found that the capacity gap for the MIMO system with MMSE and ZF receiver increases with the increase in SNR level and MMSE receiver based MIMO system provide overall better performances in compassion to $\mathrm{ZF}$ receiver based MIMO system in rich scattering environment.

\section{REFERENCES}

[1] G. J. Foschini and M. J. Gans, "On limits of wireless communications in fading environments when using multiple antennas," Wireless Pers.Commun., vol. 6, pp. 311-335, 1998.

[2] J. H. Winters, "On the capacity of radio communications systemswith diversity in rayleigh fading environments," IEEE J. Select. Areas Commun., vol. JSAC-5, pp. 871-878, June 1987.

[3] Branka Vucetic, Jinhong Yuan, "Space-Time Coding", John Wiley \& Sons Ltd, 2003.

[4] G. J. Foschini, "Layered space-time architecture for wireless communication in a fading environment when using multielement antennas", Bell Labs. Technology. Journal,Vol. 1, No.2, PP. 41-59, 1996.
[5] G. J. Foschini, "Layered space-time architecture for wireless communication", Bell Labs. Technology. Journal, Vol. 6, No.3, PP. 311-335, 1998.

[6] I.E.Telatar, "Capacity of multi-antenna Gaussian channels," Eur. Trans. Telecommun., vol.10, pp.585-595, Nov, 1999.

[7] Andrea Goldsmith, Syed Ali Jafar, Nihar Jindal, and Sriram Vishwanath, "Capacity Limits of MIMO Channels", IEEE Journal on Selected Areas in Communication, Vol.21, No.5, June, 2003.

[8] G.L.St"uber, Principles of Mobile Communication. Norwell, MA:Kluwer Academic Publishers, 1996.

[9] Li-Chun Wang, Wei-Cheng Liu, , Yun-Huai Cheng, "Capacity fades analysis of MIMO Rician channels in mobile ad hoc networks", Performance Evaluation 66, 2009742 753, Elsevier.

[10] Imdad Khan and Peter S.Hall, "Experimental Evaluation of MIMO Capacity and Correlation for Narrow band BodyCentric Wireless Channels", IEEE Transactions on Antennas and Propagation, Vol.58, No.1, January, 2010.

[11] Eko N. Onggosanusi, Anand G. Dabak, Timothy Schmidl Tarik Muharemovic, "Capacity analysis of frequencyselective MIMO channels with sub-optimal detectors", ICASSP-93.

[12] Sudharman K. Jayaweera and H.Vincent Poor, "On the capacity of multiple-antenna systems in Rician fading", IEEE Transactions on Wireless Communication, Volume: 4 Issue: 3, 1102 - 1111, May 2005

[13] Peter Drotár, Juraj Gazda, Pavol Galajda, Dušan Kocur, "Receivers for Spatially Multiplexed MIMO Transmission Systems", RTT, 2009.

[14] J. G. Proakis, Digital Communications. McGraw-Hill Inc., Third Edition, 1995. 